\title{
Harnessing the GaAs quantum dot nuclear spin bath for quantum control
}

\author{
Hugo Ribeiro, ${ }^{1}$ J. R. Petta, ${ }^{2}$ and Guido Burkard ${ }^{1}$ \\ ${ }^{1}$ Department of Physics, University of Konstanz, D-78457 Konstanz, Germany \\ ${ }^{2}$ Department of Physics, Princeton University, Princeton, NJ 08544, USA
}

\begin{abstract}
We theoretically demonstrate that nuclear spins can be harnessed to coherently control twoelectron spin states in a double quantum dot. Hyperfine interactions lead to an avoided crossing between the spin singlet state and the $m_{\mathrm{s}}=+1$ triplet state, $\mathrm{T}_{+}$. We show that a coherent superposition of singlet and triplet states can be achieved using finite-time Landau-Zener-Stückelberg interferometry. In this system the coherent rotation rate is set by the Zeeman energy, resulting in $\sim 1$ nanosecond single spin rotations. We analyze the coherence of this spin qubit by considering the coupling to the nuclear spin bath and show that $T_{2}^{*} \sim 16 \mathrm{~ns}$, in good agreement with experimental data. Our analysis further demonstrates that efficient single qubit and two qubit control can be achieved using Landau-Zener-Stückelberg interferometry.
\end{abstract}

\section{INTRODUCTION}

Considerable effort has been made in the past few years to implement qubits in nanoscale solid state structures. One of the most promising candidates are spin qubits confined in electrostatically defined quantum dots (QDs) embedded in GaAs structures.1.2. A universal set of quantum gates has been demonstrated in GaAs double quantum dots (DQD) through the achievements of single-spin rotations and the two-spin exchange interaction that generates the $\sqrt{\mathrm{SWAP}}$ gate $\mathrm{e}^{\underline{3}-\underline{\underline{5}}}$. Despite these advances, coherence times are limited by the hyperfine interaction, which couples the trapped electron spin in the quantum dot to the spin- $\frac{3}{2}$ nuclei of the GaAs substrate. The resulting nuclear fields cause rapid electron spin dephasing, leading to an inhomogenous dephasing time $T_{2}^{*} \sim 10 \mathrm{~ns}$. As each electron spin is coupled to approximately one million nuclei, the resulting behavior of the coupled electron-nuclear spin system is complicated and leads to rich dynamics that are sensitive to experimental parameters ${ }^{6}$.

The hyperfine interaction has traditionally been viewed as a nuisance. However, a recent experiment demonstrates that generation of a controlled nuclear field gradient can be used to drive fast spin rotations $\underline{\underline{7}}$. The development of quantum control methods in semiconductor quantum dots that are based on nuclear spin interactions could lead to new paradigms for single spin control.

We theoretically show that hyperfine interactions can be harnessed for quantum control in GaAs semiconductor quantum dots. In the presence of an external magnetic field $B$, which splits the triplet states, the hyperfine interaction results in an avoided crossing between the spin singlet $\mathrm{S}$ and spin triplet $\mathrm{T}_{+}$, which form the basis of a new type of spin qubit. Coherent quantum control of this qubit has already been experimentally achieved through Landau-Zener-Stückelberg (LZS) interferometry ${ }^{-}$, a technique previously used to coherently manipulate superconducting qubits 9.10 and which is based on the interference due to repeated LZS tunneling events 11 -14. The original LZS problem studies a two level system which exhibits an avoided crossing when an external control parameter is (a)

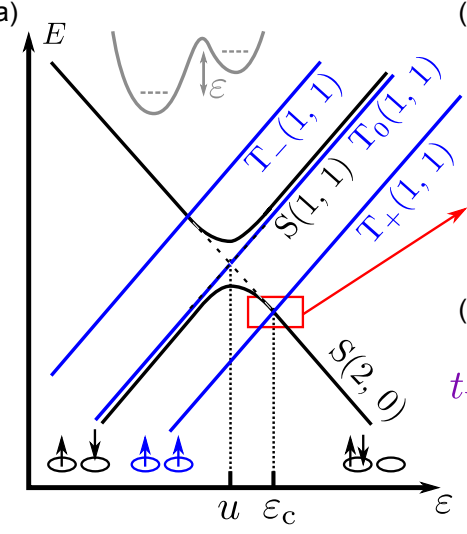

(b)

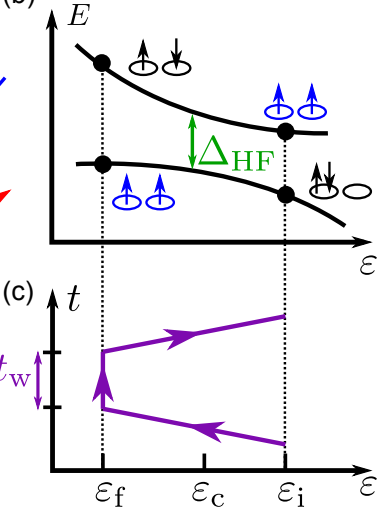

Figure 1. (color online) (a) Energy diagram for the relevant states in the DQD as a function of $\varepsilon$. The spin states for the implementation of the qubit are the hybridized singlet $\mathrm{S}$ and the triplet $\mathrm{T}_{+}$. (b) A coherent superposition of the qubit states is generated by LZS interferometry. (c) The initialized $\mathrm{S}(2,0)$ is swept through the avoided crossing by means of an applied linear gate voltage pulse $\varepsilon_{\mathrm{i}} \rightarrow \varepsilon_{\mathrm{f}}$. The final state is a coherent superposition of $\mathrm{S}$ and $\mathrm{T}_{+}$generated by LZS tunneling. At $\varepsilon_{\mathrm{f}}$, the system evolves in the external magnetic field $B$ for a time $t_{\mathrm{w}}$ before a reverse gate voltage pulse brings the system back to $\varepsilon_{\mathrm{i}}$ where a QPC measurement is performed to determine the singlet state return probability, $P_{\mathrm{S}}$.

changed. If the control parameter is time dependent, the system can be brought from an initial state through the avoided crossing. This passage may result in a change of populations and relative phase of the states. In the LZS version, the system is driven from $t_{\mathrm{i}}=-\infty \rightarrow t_{\mathrm{f}}=\infty$ and the difference in energy between the two states is a linear function of time, $\Delta E(t)=\alpha t$, which leads to the well-known result for the non-adiabatic transition probability $P_{\mathrm{LZS}}=\exp \left(-2 \pi\left|\left\langle\mathrm{S}\left|H_{\mathrm{int}}\right| \mathrm{T}_{+}\right\rangle\right|^{2} / \alpha \hbar\right)$.

For the DQD system, the usual infinite-time asymptotic theory describing LZS interferometry cannot be used. The avoided crossing originates from the hyperfine interaction between the electronic spins and the nuclear spins whose fluctuations result in a poorly defined crossing position. The phenomena observed in the exper- 
iments can only be properly described using finite-time LZS theory 15 .

To prove that our formalism describes correctly the coherent manipulation of the $\mathrm{S}-\mathrm{T}_{+}$based qubit, we will compare it to the experimentally measurable quantity $P_{\mathrm{S}}$, the singlet return probability. We then show how single qubit operations can be engineered either by the Euler angle method for rotations or by only using LZS interferometry. Finally, we demonstrate that a two qubit gate can be achieved by capacitively coupling two $\mathrm{S}-\mathrm{T}_{+}$ qubits. In contrast to the $\mathrm{S}_{-} \mathrm{T}_{0}$ qubit, where the rotation rate is set by a charge-noise-susceptible exchange energy, the rotation rate in the $\mathrm{S}-\mathrm{T}_{+}$qubit is set by the Zeeman energy and approaches $1 \mathrm{~ns}$ for modest magnetic fields of $100 \mathrm{mT}$.

\section{MODEL}

The spin preserving Hamiltonian,

$$
H_{0}=\sum_{j s} \varepsilon_{j s} n_{j s}+u \sum_{j} n_{j \uparrow} n_{j \downarrow}+\tau \sum_{s}\left(c_{1 s}^{\dagger} c_{2 s}+\text { h.c }\right),
$$

with $\varepsilon_{j s}=\varepsilon_{j}+g^{*} \mu_{\mathrm{B}} B s / 2$ and $n_{j s}=c_{j s}^{\dagger} c_{j s}$ describes the coupling between two electrons in a DQD in a magnetic field $B . g^{*}$ denotes the effective Landé g-factor ( -0.44 for GaAs), $\mu_{\mathrm{B}}$ the Bohr magneton, the $j=1,2$ and $s=\uparrow, \downarrow= \pm 1$ label the dot number and spin. The first term is the single-particle energy of the confined electrons, the second accounts for the Coulomb energy $u$ of two electrons on the same $\mathrm{QD}$, and the last for tunneling with strength $\tau$ between the dots.

The diagonalization of the first two terms of Eq. (1) leads to the relevant charge states of a DQD: the singlets $\mathrm{S}(0,2), \mathrm{S}(2,0), \mathrm{S}(1,1)$ and triplets $\mathrm{T}_{ \pm, 0}(1,1)$ where $(l, r)$ denotes the charge configuration of the dots (see Fig. 1(a)). The other states can be neglected as they have energies much higher than those considered here. The degeneracy of the singlets $\mathrm{S}(2,0)$ and $\mathrm{S}(1,1)$ at $\varepsilon= \pm u$ is lifted by the inter-dot tunneling, resulting in a splitting of $\sqrt{2} \tau$. The hyperfine interaction $H_{\mathrm{HF}}=\mathbf{S}_{1} \cdot \mathbf{h}_{1}+\mathbf{S}_{2} \cdot \mathbf{h}_{2}$ between the electron spins $\mathbf{S}_{i}$ and the nuclear spins $\mathbf{I}_{i}^{k}$ opens a splitting $\Delta_{\mathrm{HF}}$ at the degeneracy point $\varepsilon_{\mathrm{c}}$ of the singlet $\mathrm{S}$ and the triplet $\mathrm{T}_{+}$. Here, $\mathbf{h}_{\mathrm{i}}=\sum_{k=1}^{n(i)} A_{i}^{k} \mathbf{I}_{i}^{k}$ is the Overhauser (effective nuclear) field operator. The sum runs over the $n(i)$ nuclear spins in $\operatorname{dot} i$ and $A_{i}^{k}=$ $v_{i k} \nu_{0}\left|\Psi_{i}\left(\mathbf{r}_{k}\right)\right|^{2}$ is the hyperfine coupling constant with the $k$-th nucleus in $\operatorname{dot} i$, with $\Psi_{i}\left(\mathbf{r}_{k}\right)$ the electron wave function, $\nu_{0}$ the volume of the unit cell and $v_{i k}$ the hyperfine coupling strength. From now on, since we assume symmetric dots, we have $n(1)=n(2)=n$. Introducing $S_{i}^{ \pm}=S_{i}^{x} \pm \mathrm{i} S_{i}^{y}$ and $h_{i}^{ \pm}=h_{i}^{x} \pm \mathrm{i} h_{i}^{y}$, we write

$$
H_{\mathrm{HF}}=\frac{1}{2} \sum_{i}\left(2 S_{i}^{z} h_{i}^{z}+S_{i}^{+} h_{i}^{-}+S_{i}^{-} h_{i}^{+}\right) .
$$

To determine which spin states are relevant for our theory, we consider $P_{\mathrm{LZS}}$ from the asymptotic LZS model and the result of Ref. 16 for multiple level crossings to estimate the order of magnitude of the transition probabilities. The initialization of the system is done by preparing a singlet $\mathrm{S}(2,0)\left(\varepsilon_{\mathrm{i}}>\varepsilon_{\mathrm{c}}\right)$, then $\varepsilon$ is swept to achieve $\varepsilon_{\mathrm{f}}<\varepsilon_{\mathrm{c}}$. During this operation, the system goes through three avoided crossings (cf Fig. 1). To estimate the matrix element $\left|\left\langle\mathrm{S}\left|H_{\mathrm{HF}}\right| \mathrm{T}_{+}\right\rangle\right|^{2}=\left|\left\langle\mathrm{S}\left|H_{\mathrm{HF}}\right| \mathrm{T}_{-}\right\rangle\right|^{2}=\left|\Delta_{\mathrm{HF}}\right|^{2}$, we use the experimentally found value of $\Delta_{\mathrm{HF}}=60 \mathrm{neV}$ from Ref. 8. The matrix element entering $P_{\text {LZS }}$ at the avoided crossing between $\mathrm{S}(2,0)$ and the excited singlet state $S^{\prime}(1,1)$ is given by $\left|\left\langle S(2,0)\left|H_{0}\right| S^{\prime}(1,1)\right\rangle\right|^{2}=2 \tau^{2}$, with $\tau=5 \mu \mathrm{eV}$. The order of magnitude of $\alpha$ is taken between $10^{-3}-10^{-2} \mathrm{meV} / \mathrm{ns}$. We find $P_{\mathrm{T}_{+}} \simeq 0.97-0.99$ and $P_{\mathrm{S}^{\prime}(1,1)}, P_{\mathrm{T}_{-}} \ll 10^{-8}$. These results show that population of the excited singlet and $\mathrm{T}_{-}$level are negligible. $\mathrm{T}_{0}$ can also be neglected because it does not cross with any other level, it splits from $\mathrm{S}(1,1)$ due to the exchange coupling 22 .

Near the $\mathrm{S}-\mathrm{T}_{+}$crossing, the dynamics can be restricted to the Hilbert space spanned by $\mathrm{T}_{+}(1,1), \mathrm{S}(1,1)$, and $\mathrm{S}(2,0)$ and described by

$$
H_{\mathrm{S}, \mathrm{T}_{+}} \simeq\left(\begin{array}{ccc}
g^{*} \mu_{\mathrm{B}} B & 0 & 0 \\
0 & 0 & \sqrt{2} \tau \\
0 & \sqrt{2} \tau & u-\varepsilon
\end{array}\right)
$$

where we can neglect an additive term $\propto \varepsilon \mathbb{1}$, with $\varepsilon=\varepsilon_{1}-\varepsilon_{2}$ the detuning of the dots. According to our previous estimate, we can reduce Eq. (3) to a $2 \times 2$ Hamiltonian which only takes into account the lowest hybridized singlet $|\mathrm{S}\rangle=c(\varepsilon)|\mathrm{S}(1,1)\rangle+$ $\sqrt{1-c(\varepsilon)^{2}}|\mathrm{~S}(2,0)\rangle$ and the triplet $\left|\mathrm{T}_{+}\right\rangle=\left|\mathrm{T}_{+}(1,1)\right\rangle$, where $c(\varepsilon)=(-u+\varepsilon-\eta) / \sqrt{8 \tau^{2}+(u-\varepsilon+\eta)^{2}}$, with $\eta=\sqrt{8 \tau^{2}+(u-\varepsilon)^{2}}$. The energy associated with the lowest hybridized singlet is $E_{\mathrm{S}}(\varepsilon)=(u-\varepsilon-\eta) / 2$ and the energy of the triplet is $E_{\mathrm{T}_{+}}=g^{*} \mu_{\mathrm{B}} B$. The Hamiltonian describing the dynamics of the lowest energy states in the vicinity of $S-T_{+}$can therefore be written as

$$
H_{0}(\varepsilon)=E_{\mathrm{S}}(\varepsilon)|\mathrm{S}\rangle\left\langle\mathrm{S}\left|+E_{\mathrm{T}_{+}}\right| \mathrm{T}_{+}\right\rangle\left\langle\mathrm{T}_{+}\right| .
$$

Another relevant quantity derived from Eq. (3) is the degeneracy position $\varepsilon_{\mathrm{c}}$ of $\mathrm{S}$ and $\mathrm{T}_{+}$, given by the funnelshaped function

$$
\varepsilon_{\mathrm{c}}(u, B)=u+2 \tau^{2} / g \mu_{\mathrm{B}} B-g \mu_{\mathrm{B}} B .
$$

\section{SEMI-CLASSICAL THEORY}

We model the Overhauser field classically, such that it acts on the electron spin as a magnetic field $\mathbf{B}_{\mathrm{n}, i}$ with $\mathbf{h}_{i}=g^{*} \mu_{\mathrm{B}} \mathbf{B}_{\mathrm{n}, i}$ and its physical properties are given by a statistical distribution that reflects the quantum fluctuations of the nuclear ensemble. At typical operating temperatures and external magnetic fields $k_{B} T \gg g_{\mathrm{N}} \mu_{\mathrm{N}} B$, where $g_{\mathrm{N}}$ and $\mu_{\mathrm{N}}$ are the nuclear $g$ factor and magneton. In this limit we can assume the nuclei to be completely unpolarized, resulting in a Gaussian distribution 
of nuclear fields $17,18 p\left(B_{\mathrm{n}, i}\right)=(1 / \sqrt{2 \pi} \sigma) \mathrm{e}^{-B_{\mathrm{n}, i}^{2} / 2 \sigma^{2}}$, with $\sigma=A / g^{*} \mu_{\mathrm{B}} \sqrt{n}$ and $A \approx 90 \mu \mathrm{eV}$. The effective Hamiltonian describing the qubit dynamics around the $\mathrm{S}_{-} \mathrm{T}_{+}$ avoided crossing is given by

$$
H_{\mathrm{eff}}(\varepsilon)=H_{0}(\varepsilon)+\frac{1}{2} g^{*} \mu_{\mathrm{B}} \sum_{i}\left(S_{i}^{+} B_{\mathrm{n}, i}^{-}+S_{i}^{-} B_{\mathrm{n}, i}^{+}\right) .
$$

We include the nuclear Zeeman spliting in the $\mathrm{T}_{+}$energy, $E_{\mathrm{T}_{+}}=g^{*} \mu_{\mathrm{B}}\left(B+B_{\mathrm{n}}^{z}\right)$, where $B_{\mathrm{n}}^{z}=B_{\mathrm{n}, 1}^{z}+B_{\mathrm{n}, 2}^{z}$ and $B_{\mathrm{n}, i}^{ \pm}=B_{\mathrm{n}, i}^{x} \pm \mathrm{i} B_{\mathrm{n}, i}^{y}$. The classical approximation of the Overhauser fields is possible because the nuclear state changes only slightly after a single sweep $\underline{19}$. To obtain an analytical expression of the LZS propagator (see Appendix A), we linearize the difference in energy $\Delta E(t)$ around $t=0 \underline{15}$ by assuming $\varepsilon(t)=\gamma t+\varepsilon_{\mathrm{c}}$, we find

$$
\alpha=\frac{\left(g \mu_{\mathrm{B}}\left(B+B_{\mathrm{n}}^{z}\right)\right)^{2}}{2 \tau^{2}+\left(g \mu_{\mathrm{B}}\left(B+B_{\mathrm{n}}^{z}\right)\right)^{2}} \gamma .
$$

Here $\gamma$ is the rate at which the external voltage gates are ramped. Control over $\alpha$ can therefore be achieved by modifying $\gamma$.

To test our model we first compute the singlet return probability $P_{\mathrm{S}}$, an experimentally observable quantity, as a function of the final detuning $\varepsilon_{\mathrm{f}}$ and waiting time $t_{\mathrm{w}}$,

$$
P_{\mathrm{S}}=\int \prod_{k=1,2} \mathrm{~d} \mathbf{B}_{\mathrm{n}, k} p\left(\mathbf{B}_{\mathrm{n}, k}\right)\left|\left\langle\mathrm{S}\left|U\left(\mathbf{B}_{\mathrm{n}, 1}, \mathbf{B}_{\mathrm{n}, 2}\right)\right| \mathrm{S}\right\rangle\right|^{2},
$$

with

$$
U\left(\mathbf{B}_{\mathrm{n}, 1}, \mathbf{B}_{\mathrm{n}, 2}\right)=U_{\mathrm{b}}\left(\mathbf{B}_{\mathrm{n}, 1}, \mathbf{B}_{\mathrm{n}, 2}\right) U_{\mathrm{w}}\left(B_{\mathrm{n}}^{z}\right) U_{\mathrm{f}}\left(\mathbf{B}_{\mathrm{n}, 1}, \mathbf{B}_{\mathrm{n}, 2}\right),
$$

where $U_{\mathrm{b}, \mathrm{f}}=\mathrm{T} \exp \left[-\mathrm{i} \int_{t_{\mathrm{i}}}^{t_{\mathrm{f}}} \mathrm{d} t H_{\mathrm{eff}}(\varepsilon(t)) / \hbar\right]$ are the backward and forward LZS propagators and $U_{\mathrm{w}} \simeq$ $\mathrm{T} \exp \left[-\mathrm{i} \int_{0}^{t_{\mathrm{w}}} \mathrm{d} t H_{0}\left(\varepsilon_{\mathrm{f}}\right) / \hbar\right]$ describes the evolution of the system during the waiting time $t_{\mathrm{w}}$ at the final detuning position $\varepsilon_{\mathrm{f}}$ with $\left|\varepsilon_{\mathrm{f}}-\varepsilon_{\mathrm{c}}\right| \gtrsim \Delta_{\mathrm{HF}}$. We evaluate $P_{\mathrm{S}}$ by numerical sampling. Instead of estimating $\sigma$ from $n$ and $A$ of the QDs, here we use the experimentally determined ${ }^{8}$ $\Delta_{\mathrm{HF}}=60 \mathrm{neV}$ to derive $\sigma \sim 1.67 \mathrm{mT}$. We have $\left\langle\Delta_{\mathrm{HF}}^{2}\right\rangle=$ $\left\langle\left(\delta B_{\mathrm{n}}^{-}\right)^{2}\right\rangle=4 \sigma^{2}$, with $\delta B_{\mathrm{n}}^{-} \equiv g^{*} \mu_{\mathrm{B}}\left(B_{\mathrm{n}, 2}^{-}-B_{\mathrm{n}, 1}^{-}\right) / 2 \sqrt{2}=$ $\left\langle\mathrm{T}_{+}\left|H_{\mathrm{HF}}^{\perp}\right| \mathrm{S}\right\rangle$. In Fig. 2 we show $P_{\mathrm{S}}$ as a function of $\varepsilon_{\mathrm{f}}$ and $t_{\mathrm{w}}$ for $\gamma=0.015 \mathrm{meV} / \mathrm{ns}, B=100 \mathrm{mT}, u=4 \mathrm{meV}$, and $\tau=5 \mu \mathrm{eV}$. We use a square pulse with a ramping time $\left|t_{\mathrm{f}}-t_{\mathrm{i}}\right|$ fixed to $1.5 \mathrm{~ns}$ and the initial detuning $\varepsilon_{\mathrm{i}}$ is varied to reach different values of $\varepsilon_{\mathrm{f}}$.

We identify coherent oscillations as a function of $t_{\mathrm{w}}$ and $\varepsilon_{\mathrm{f}}$. From a best fit, we obtain the decoherence time $T_{2}^{*}=(16.0 \pm 0.4) \mathrm{ns}$, which agrees well with experiment. The decoherence is mainly due to the fluctuations of $B_{\mathrm{n}}^{z}$. The period of the temporal oscillations is $T=h /\left|E_{\mathrm{S}}\left(\varepsilon_{\mathrm{f}}\right)-E_{\mathrm{T}_{+}}\right| \sim 4.3 \mathrm{~ns}$ for $\varepsilon_{\mathrm{f}}=3.97 \mathrm{meV}$ (see Fig. 2(b)). For a fixed $B$, a shorter period can be obtained for smaller $\varepsilon_{\mathrm{f}}$, the fastest oscillations being defined by the Zeeman energy. To further decrease the period the

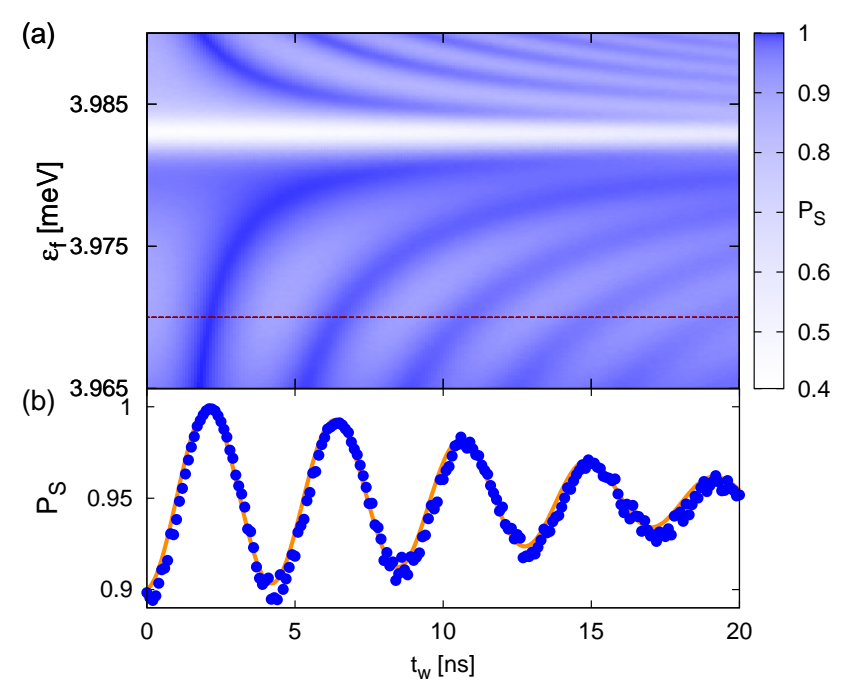

Figure 2. (color online) Theoretical results. (a) The singlet return probability $P_{\mathrm{S}}$ from the semi-classical model as a function of the waiting time $t_{\mathrm{w}}$ and final detuning $\varepsilon_{\mathrm{f}}$. We find nanosecond oscillation periods and the dephasing time $T_{2}^{*} \sim 16 \mathrm{~ns}$, in good agreement with experiment. We used $B=100 \mathrm{mT}, \gamma=0.015 \mathrm{meV} / \mathrm{ns}, u=4 \mathrm{meV}$ and $\tau=5 \mu \mathrm{eV}$. (b) $P_{\mathrm{S}}$ (blue) as a function of $t_{\mathrm{w}}$ for $\varepsilon_{\mathrm{f}}=3.97 \mathrm{meV}$ (horizontal line in (a)). We plot (orange) $C \sin (\omega t) \exp \left(-\left(t / T_{2}^{*}\right)^{2}\right)$ where $\omega=\left|E_{\mathrm{S}}\left(\varepsilon_{\mathrm{f}}\right)-E_{\mathrm{T}_{+}}\right| / \hbar \sim 2 \pi \cdot 0.23 \mathrm{GHz}, C=0.95$ and $T_{2}^{*}=(16.0 \pm 0.4) \mathrm{ns}$ is extracted from a best fit.

external $B$ field can be increased and hence the qubit manipulation could be done in a time scale of $100 \mathrm{ps}$ for $B \sim 1.6 \mathrm{~T}$, which would allow $\sim 160$ coherent operations within $T_{2}^{*}$. In the exchange gate demonstrated in Ref. 4, $\mathrm{d} J / \mathrm{d} \varepsilon$ increases with $\varepsilon$, which results in faster dephasing for faster rotations. In contrast, here the rotation rate is set by the Zeeman energy, which is independent of $\varepsilon$ far from the $\mathrm{S}-\mathrm{T}_{+}$avoided crossing. As a result, the coherent oscillation frequency can be increased without making the qubit more susceptible to gate voltage fluctuations by simply increasing $B$. Far from the avoided crossing the level spacing is independent of detuning, similar to the "sweet spot" in superconducting qubits 20 .

The model predicts coherent oscillations in $P_{\mathrm{S}}$ for $\varepsilon_{\mathrm{f}}>\varepsilon_{\mathrm{c}}$, i.e. in the case where the qubit has not passed the avoided crossing. It can be explained within finitetime LZS theory, but not with the conventional asymptotic Landau-Zener formula. In other words, even if $t_{\mathrm{f}}<0$, we have $\left|U_{i j}^{\mathrm{LZS}}\left(t_{\mathrm{f}}, t_{\mathrm{i}}\right)\right|^{2}>0$, which illustrates the non-adiabatic character of the problem. For the pulse conditions used in Ref. 8, oscillations are not observed for $\varepsilon_{\mathrm{f}}>\varepsilon_{\mathrm{c}}$, most likely due to charge dephasing. The coherence time of an admixture of $(1,1)$ and $(2,0)$ charge state has been measured to be $\sim 1 \mathrm{~ns}$ for GaAs $\mathrm{QDs}^{21}$ and sets the time scale at which the system must be driven to observe oscillations for $\varepsilon_{\mathrm{f}}>\varepsilon_{\mathrm{c}}$. A finite-time effect in agreement with the experimental data is the dependence of the oscillation amplitude $P_{\mathrm{S}}$ on the pulse length. Finally, we show in Fig. 3 a comparison between experiment 


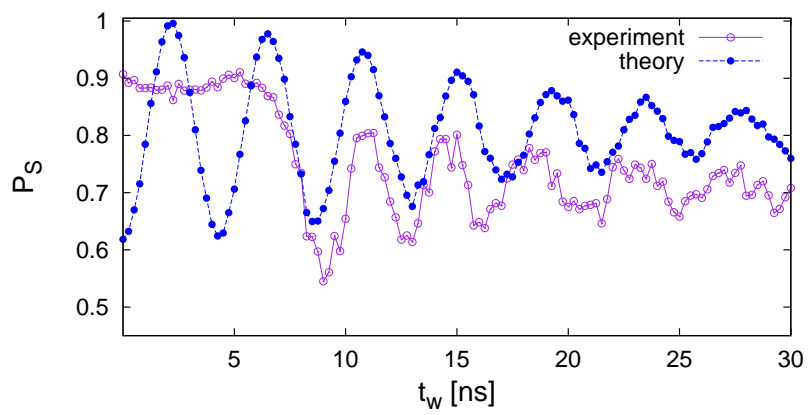

Figure 3. (color online) Comparison between experimental (purple, open circles) and theoretical values (blue, filled circles) of $P_{\mathrm{S}}$ for $B=45 \mathrm{mT}$. Theoretical values are obtained by finding the detuning $\varepsilon_{\mathrm{f}}=3.78 \mathrm{meV}$ for which the theoretical and experimental oscillation periods match. A suitable $\gamma=0.12 \mathrm{meV} / \mathrm{ns}$ is then chosen such that $\varepsilon_{\mathrm{i}}>\varepsilon_{\mathrm{c}}$. The experiments used passive filtering of square pulses to reduce $P_{\text {LzS }}$ and thereby increase the visibility of the oscillations. As a result, oscillations in the experimental data are delayed to longer times due to finite rise time effects. The theory points are obtained with a perfect square pulse.

and theory for $B=45 \mathrm{mT}$. The experimental data were obtained from the setup used in Ref. 8. The experiments used passive filtering of square pulses to reduce $P_{\text {LZS }}$ and thereby increase the visibility of the oscillations. As a result, oscillations in the experimental data are delayed to longer times due to finite rise time effects.

\section{ARBITRARY SINGLE QUBIT ROTATIONS}

The passage through the avoided crossing can be interpreted as a rotation (see Appendix C), $U_{\mathrm{LZS}}\left(\eta, t_{\mathrm{f}}, t_{\mathrm{i}}\right)=$ $\mathrm{e}^{-\mathrm{i} \sigma \cdot \hat{\mathbf{n}} \theta / 2}$ by an angle $\theta$ around the axis $\hat{\mathbf{n}}$, see Fig. 4 , Here $\theta=\theta\left(\eta, t_{\mathrm{f}}, t_{\mathrm{i}}\right)$ and $\hat{\mathbf{n}}=\hat{\mathbf{n}}\left(\eta, t_{\mathrm{f}}, t_{\mathrm{i}}\right)$ is a unit vector where $\eta=\left|\left\langle\mathrm{T}_{+}\left|H_{\mathrm{HF}}\right| \mathrm{S}\right\rangle\right| / \sqrt{\alpha \hbar}$ is the dimensionless coupling strength. Since $\hat{n}$ and $\theta$ are functions of the same experimental parameters $t_{\mathrm{i}}\left(\rightarrow \varepsilon_{\mathrm{i}}\right), t_{\mathrm{f}}\left(\rightarrow \varepsilon_{\mathrm{f}}\right)$, and $\alpha(\rightarrow \gamma)$, it is not straight forward to find them simultaneously in order to build a given single qubit rotation. For instance, fixing two parameters and tuning the third one will simultaneously change $\hat{n}$ and $\theta$ limiting the achievable rotations angles. Nevertheless, the situation is not hopeless and several composite methods can be engineered to achieve any rotation. We present here three methods, each of them having their own advantages.

Since rotations by an angle $\varphi$ around the $z$-axis are available by letting the qubit evolve in an external $B$ field, we would like $\hat{\mathbf{n}}$ to be in the $x y$-plane in order to build any rotation by the Euler angle method. Below we show that this is possible if for example (see Appendix C) the propagation times are equal, $-t_{\mathrm{i}}=t_{\mathrm{f}}=t_{\mathrm{LZS}}$. However, a $\pi$-rotation from $|S\rangle$ to $\left|\mathrm{T}_{+}\right\rangle$would take an exponentially long time with a single LZS transition, since it corresponds to a fully adiabatic transition. However,
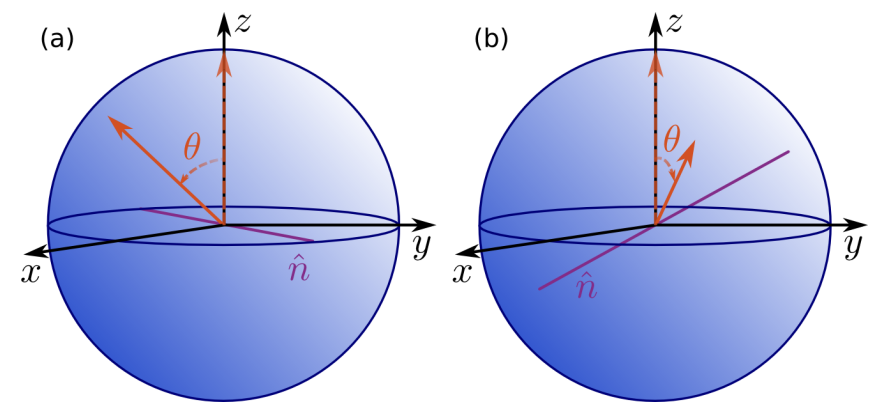

Figure 4. (color online) Bloch sphere representation of LZS transitions as rotations. (a) When the propagation times are equal, $-t_{\mathrm{i}}=t_{\mathrm{f}}$, the rotation axis lays in the $x y$-plane. In addition with the rotations generated around the $z$-axis by letting the qubit evolve in the external magnetic field, any rotation can be achieved by the Euler angle method. (b) If the propagation times are different the rotations axis may not lay (see Appedix ) in the $x y$ plane. In this case, a pure LZS interferometry technique can be used to generate any rotation.

this problem can be circumvented by sequentially applying several LZS transitions. A $\pi$-rotation from $|S\rangle$ to $\left|T_{+}\right\rangle$ can be achieved in $\sim 0.1-1.0$ ns for two consecutive and identical LZS transitions.

The single qubit gates can also be implemented with a pure LZS interferometry technique, similar to the one used to control superconducting qubits. This method requires sequential driving of the qubit through the avoided crossing. The different passages result in a series of LZS transitions each of them corresponding to a rotation of the qubit. By tuning $\gamma$ and choosing $-t_{\mathrm{i}} \neq t_{\mathrm{f}}$ with different ratios for $\left|t_{\mathrm{i}}\right| /\left|t_{\mathrm{f}}\right|$, any qubit rotations can be achieved within a nanosecond.

Since finite-time effects are present in the system, we can think about a control method where the qubit is operated on the $(1,1)$ charge configuration side ("sweet region"). This requires the preparation of a $T_{+}(1,1)$ state $^{7}$ and pulses with rise times shorter than $1 \mathrm{~ns}$ which do not drive the system through the avoided crossing unless a measurement is required. The qubit manipulation is achieved through finite-time LZS interferometry, where tuning $\gamma$ and the propagation time $t_{\mathrm{i}, \mathrm{f}}$ allows to achieve any desirable angle. For all those methods, an arbitrary single qubit rotation can be expressed as a series of a forward sweep -wait -backward sweep operators,

$$
\mathcal{D}(\theta, \varphi)=\prod_{i=1}^{l} U_{\mathrm{b}}\left(\theta_{\mathrm{b}}^{(i)}\right) U_{z}\left(\varphi^{(i)}\right) U_{\mathrm{f}}\left(\theta_{\mathrm{f}}^{(i)}\right)
$$

which reduces to Eq. (8) for $l=1$. The proposed methods require a maximum of $l=3$. It is important to notice that the rotation axis and the final measurable angles will not be $\hat{\mathbf{n}}, \theta=\sum_{i} \theta_{i}$, and $\varphi=\sum_{i} \varphi_{i}$, but rather $\langle\hat{\mathbf{n}}\rangle_{\chi}$, $\langle\theta\rangle_{\chi}$, and $\langle\varphi\rangle_{\chi}$, where the brackets denote the averaging over the nuclear spin bath. A similar scheme with $l=1$ has been proposed for the $\mathrm{S}-\mathrm{T}_{0}$ qubit $^{22}$. 

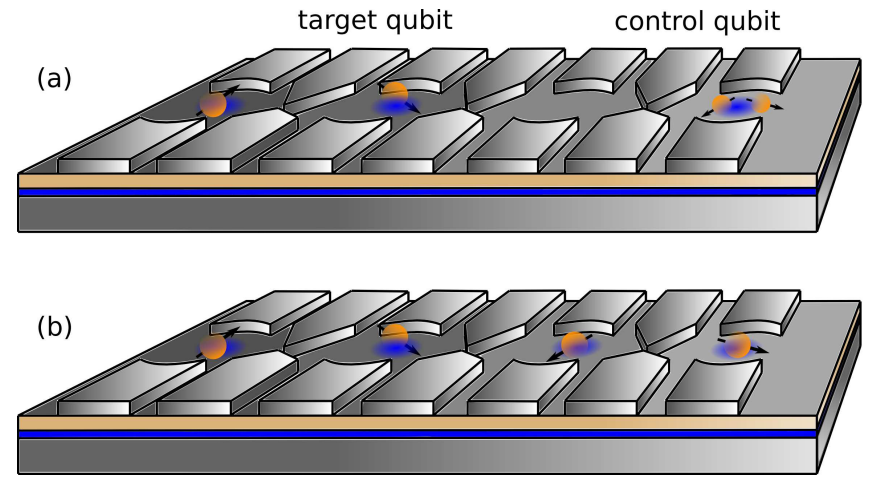

Figure 5. (color online) A conditional gate can be implemented by capacitively coupling electrons trapped in quantum dots belonging to different qubits. The crossing position $\varepsilon_{\mathrm{c}}$ has different values whenever the charge state of the control qubit is $(02)(a)$ or $(1,1)(b)$. The later case results in $\varepsilon_{\mathrm{f}} \ll \varepsilon_{\mathrm{c}}$ which suppresses any LZS transition.

\section{TWO QUBIT GATE}

To complete the set of quantum gates, a two-qubit operation such as CNOT is required. We consider the Hamiltonian

$$
H_{\mathrm{eff}}^{(1)}+H_{\mathrm{eff}}^{(2)}+H_{\mathrm{int}},
$$

where $H_{\mathrm{eff}}^{(i)}$ is the single qubit Hamiltonian (6) and $H_{\mathrm{int}}=$ $\tilde{u} \sum_{j=2,3} n_{j \uparrow} n_{j \downarrow}$ describes the capacitive coupling ${ }^{22-24}$ between two adjacent QDs belonging to different $\mathrm{S}-\mathrm{T}_{+}$ qubits. Tunneling between the dots of the different qubits can be suppressed by an appropriate gate voltage. If the control qubit, qubit- 1 , is in a $\mathrm{S}(2,0)$ state, $H_{\text {int }}=0$ and the dynamics of the target qubit, qubit-2, is reduced to the case of a single qubit, see Fig. 5. When the control qubit is in a $(1,1)$ charge configuration, the target qubit is influenced by the interdot Coulomb interaction. In this case, the dynamics of the target qubit can be described by Eq. (6) by replacing $u \rightarrow u+\tilde{u}$. In particular, this affects the position of the avoided crossing. For a system with two DQD separated by a distance $d \simeq 2 R-10 R$, where $R$ is the approximate radius of one QD, the intradot Coulomb interaction $u \sim e^{2} / R$ is comparable to the interdot Coulomb interaction $\tilde{u} \sim e^{2} / d$ resulting in $\left|\varepsilon_{\mathrm{c}}(u)-\varepsilon_{\mathrm{c}}(u+\tilde{u})\right|>1 \mathrm{meV}$. From the previous discussion, we know that a $\pi$-rotation is possible within $\sim 1 \mathrm{~ns}$ if $\varepsilon_{\mathrm{c}}=\varepsilon_{\mathrm{c}}(u)$. In the case where the avoided crossing is at $\varepsilon_{\mathrm{c}}(u+\tilde{u})$ the same LZS sequence will leave the target qubit unchanged, even within a finite-time theory since the separation between the two avoided crossings is $>1 \mathrm{meV}$. Therefore, we estimate the CNOT gate time to be $\sim 1-3$ ns.

Let us consider the case where the control qubit is in the $S(2,0)$ state, which is the logical $|1\rangle$ of the qubit, such that the target and control qubit are not capacitively coupled, $u=4 \mathrm{meV}$. For $\gamma=0.3 \frac{\mathrm{meV}}{\mathrm{ns}}, B=100 \mathrm{mT}$, $\varepsilon_{\mathrm{i}}=3.98597 \mathrm{meV}$, and $\varepsilon_{\mathrm{f}}=3.97991 \mathrm{meV}$ we find for $l=6$ and $\varphi^{(i)}=0$ in Eq. (10)

$$
\mathcal{D}^{(1)}(\theta, \varphi) \simeq\left(\begin{array}{cc}
-0.314 & 0.945-0.096 \mathrm{i} \\
-0.945-0.096 \mathrm{i} & -0.314
\end{array}\right)
$$

This example shows the almost perfect realization of a conditional $\mathrm{i} \sigma_{y}$ operation which corresponds to a CNOT gate up to single-qubit gates.

To show that this method produces a CNOT gate, we consider the case where the control qubit is in the $\mathrm{T}_{+}(1,1)$ state, which is the logical $|0\rangle$ of the qubit. We estimate a lower bound for the strength of the capacitive coupling between the qubits to be $u+\tilde{u}=5 \mathrm{meV}$ (see above). In this case, the target qubit evolution takes the form

$$
\mathcal{D}^{(0)}(\theta, \varphi) \simeq\left(\begin{array}{cc}
0.971+0.002 \mathrm{i} & -0.206-0.124 \mathrm{i} \\
0.206-0.124 \mathrm{i} & 0.971-0.002 \mathrm{i}
\end{array}\right)
$$

which is close to $\mathbb{1}$ and demonstrates the possibility of generating a CNOT gate with the proposed method.

Notice that our choice for $\varepsilon_{\mathrm{i}, \mathrm{f}}$ corresponds to a propagation time $t_{\mathrm{LZS}}=0.01 \mathrm{~ns}$ such that the total gate time to achieve controlled-i $\sigma_{y}$ is $0.24 \mathrm{~ns}$. The fidelity is $\mathcal{F}=\left|\operatorname{Tr} \mathcal{D}^{(0)}+\operatorname{Tr}\left[\mathcal{D}^{(1)}\left(\mathrm{i} \sigma_{y}\right)^{\dagger}\right]\right|^{2} / 16 \simeq 0.918$. A more accurate CNOT gate can be engineered by fine tuning the parameters entering the LZS propagator.

\section{CONCLUSIONS}

We have demonstrated that coherent control of the S$\mathrm{T}_{+}$qubit can be achieved using LZS interferometry. Hyperfine interactions lead to an avoided crossing between $\mathrm{S}$ and $\mathrm{T}_{+}$states, which allows for efficient quantum control. Moreover, we predict that in the limit of fast risetime pulses coherent oscillations in $P_{\mathrm{S}}$ should be observed even without going through the avoided crossing. This phenomenon is a finite time effect which we have theoretically described using the general finite-time LZS theory and it can be used to operate the qubit in the $(1,1)$ charge configuration side ("sweet region").

Our scheme can be extended to DQD in materials with few nuclear spins (graphene, CNT, Si). In such cases, the avoided crossing between the qubit states can be achieved by engineering a DQD in the presence of micromagnets which provide the in-plane gradient magnetic field for the realization of the LZS based gates ${ }^{25}$. The qubit will moreover benefit from the lack of the inhomogeneous broadening due to the Overhauser fields and exhibit an extended $T_{2}^{*}$. In GaAs DQDs the method proposed in Ref. 7 could be used to extend $T_{2}^{*}$ without cancelling the gradient field. Other schemes to polarize the nuclear spins ${ }^{26}$ or reduce their fluctuations 27,28 also exist. 


\section{ACKNOWLEDGMENTS}

We acknowledge funding from the DFG within SPP 1285, FOR 912 and SFB 767. Research at Princeton was supported by the Sloan and Packard Foundations, DARPA award N66001-09-1-2020, and the NSF through DMR-0819860 and DMR-0846341.

\section{Appendix A: The Landau-Zener-Stückelberg finite-time propagator}

In this appendix we follow the work of Vitanov and Garraway $\frac{15}{5}$ and consider, without loss of generality, a two-level system whose eigenenergies $E_{1}$ and $E_{2}$ are time dependent, $E_{1}=E_{1}(t), E_{2}=E_{2}(t)$ and their difference is a linear function of time $2 \Delta(t)=E_{2}(t)-E_{1}(t)=\alpha t$. Furthermore, we assume the levels to be coupled with strength $\lambda$. The matrix representation of the system's Hamiltonian is given by

$$
H_{\mathrm{LZS}}(t)=\left(\begin{array}{cc}
-\Delta(t) & \lambda \\
\lambda & \Delta(t)
\end{array}\right) .
$$

The time evolution of such a system is described by the time-dependent Schrödinger equation

$$
\mathrm{i} \hbar \frac{\mathrm{d}}{\mathrm{d} t}|\psi(t)\rangle=H_{\mathrm{LZS}}(t)|\psi(t)\rangle
$$

with $|\psi(t)\rangle=c_{1}(t)|1\rangle+c_{2}(t)|2\rangle$. After substitution of (A1) into Eq. (A2), a coupled system of first order ordinary differential equations is obtained

$$
\begin{aligned}
& \mathrm{i} \hbar \dot{c}_{1}(t)=-\Delta(t) c_{1}(t)+\lambda c_{2}(t), \\
& \mathrm{i} \hbar \dot{c}_{2}(t)=\lambda c_{1}(t)+\Delta(t) c_{2}(t) .
\end{aligned}
$$

By deriving Eq. A3 with respect to time and substituting Eqs. (A3) and (A4) into the newly obtained ordinary second order differential equation, we obtain

$$
\ddot{c}_{1}(t)=\left(\frac{\mathrm{i}}{\hbar} \alpha-\frac{\alpha^{2} t^{2}}{\hbar^{2}}-\frac{\lambda^{2}}{\hbar^{2}}\right) c_{1}(t) .
$$

It is convenient to introduce dimensionless parameters before solving Eq. A5), here we introduce the dimensionless time $\tau=\sqrt{\frac{\alpha}{\hbar}} t$ which we substitute in Eq. A5 to obtain

$$
\frac{\mathrm{d}^{2}}{\mathrm{~d} \tau^{2}} c_{1}(\tau)+\left(-\mathrm{i}+\eta^{2}+\tau^{2}\right) c_{1}(\tau)=0,
$$

where $\eta=\frac{\lambda}{\sqrt{\alpha \hbar}}$ is the dimensionless coupling strength.

The solution of Eq. (A6) is

$$
c_{1}(t)=\kappa_{1} D_{\frac{\mathrm{i} \eta^{2}}{2}}\left(\sqrt{2} \mathrm{e}^{-\frac{\mathrm{i} \pi}{4}} \tau\right)+\kappa_{2} D_{\frac{\mathrm{i} \eta^{2}}{2}}\left(\sqrt{2} \mathrm{e}^{\frac{3 \mathrm{i} \pi}{4}} \tau\right),
$$

where $D_{\nu}(z)$ are parabolic cylinder functions, which solve the Weber equation 29

$$
\frac{\mathrm{d}^{2}}{\mathrm{~d} z^{2}} D_{\nu}(z)+\left(\nu+\frac{1}{2}-\frac{1}{4} z^{2}\right) D_{\nu}(z)=0,
$$

and can be obtained from Eq. A6 by writing the expression in brackets as $-2 \mathrm{i}\left(\mathrm{i} \eta^{2} / 2+1 / 2+\mathrm{i} \tau^{2} / 2\right)$ and substituting $\tau \rightarrow 2^{-1 / 2} \exp (\mathrm{i} \pi / 4) z$.

$c_{2}(t)$ is obtained by inserting Eq. (A7) into Eq. (A3) and using the property

$$
\frac{\mathrm{d}}{\mathrm{d} z}\left(\mathrm{e}^{\frac{z^{2}}{4}} D_{\nu}(z)\right)=\nu \mathrm{e}^{\frac{z^{2}}{4}} D_{\nu-1}(z) .
$$

One finds

$$
\begin{aligned}
c_{2}(t)=\frac{\eta}{\sqrt{2}} \mathrm{e}^{-\frac{\mathrm{i} \pi}{4}} & {\left[-\kappa_{1} D_{\frac{\mathrm{i} \eta^{2}}{2}-1}\left(\sqrt{2} \mathrm{e}^{-\frac{\mathrm{i} \pi}{4}} \tau\right)\right.} \\
& \left.+\kappa_{2} D_{\frac{\mathrm{i} \eta^{2}}{2}-1}\left(\sqrt{2} \mathrm{e}^{\frac{3 \mathrm{i} \pi}{4}} \tau\right)\right] .
\end{aligned}
$$

To find the constants $\kappa_{1}$ and $\kappa_{2}$, we consider initial conditions given by $c_{1}\left(\tau_{\mathrm{i}}\right)$ and $c_{2}\left(\tau_{\mathrm{i}}\right)$ and the Wronskian relation

$$
\begin{aligned}
\mathrm{W}\left\{D_{\nu}(z), D_{\nu}(-z)\right\} & :=D_{\nu}(z) \frac{\mathrm{d}}{\mathrm{d} z} D_{\nu}(-z)-D_{\nu}(-z) \frac{\mathrm{d}}{\mathrm{d} z} D_{\nu}(z) \\
& =\frac{\sqrt{2 \pi}}{\Gamma(-\nu)} .
\end{aligned}
$$

We solve the system of equation given by Eqs. (A7) and (A10) for $\kappa_{1}$ and $\kappa_{2}$ using the Wronskian property (A11), we find

$$
\begin{aligned}
\kappa_{1}= & \frac{\Gamma\left(1-\frac{\mathrm{i} \eta^{2}}{2}\right)}{\sqrt{2 \pi}}\left[D_{\frac{\mathrm{i} \eta^{2}}{2}-1}\left(\sqrt{2} \mathrm{e}^{\frac{3 \mathrm{i} \pi}{4}} \tau_{\mathrm{i}}\right) c_{1}\left(\tau_{\mathrm{i}}\right)\right. \\
& \left.-\frac{\sqrt{2}}{\omega} \mathrm{e}^{\frac{\mathrm{i} \pi}{4}} D_{\frac{\mathrm{i} \eta^{2}}{2}}\left(\sqrt{2} \mathrm{e}^{\frac{3 \mathrm{i} \pi}{4}} \tau_{\mathrm{i}}\right) c_{2}\left(\tau_{\mathrm{i}}\right)\right], \\
\kappa_{2}= & \frac{\Gamma\left(1-\frac{\mathrm{i} \eta^{2}}{2}\right)}{\sqrt{2 \pi}}\left[D_{\frac{\mathrm{i} \eta^{2}}{2}-1}\left(\sqrt{2} \mathrm{e}^{-\frac{\mathrm{i} \pi}{4}} \tau_{\mathrm{i}}\right) c_{1}\left(\tau_{\mathrm{i}}\right)\right. \\
& \left.+\frac{\sqrt{2}}{\omega} \mathrm{e}^{\frac{\mathrm{i} \pi}{4}} D_{\frac{\mathrm{i} \eta^{2}}{2}}\left(\sqrt{2} \mathrm{e}^{-\frac{\mathrm{i} \pi}{4}} \tau_{\mathrm{i}}\right) c_{2}\left(\tau_{\mathrm{i}}\right)\right] .
\end{aligned}
$$

Substituting Eqs. (A12) and (A13) into Eqs. (A7) and (A10) and having in mind that we are looking for the evolution operator $U\left(t_{\mathrm{f}}, t_{\mathrm{i}}\right)$ giving the final state knowing the initial one

$$
\left|\psi\left(t_{\mathrm{f}}\right)\right\rangle=U\left(t_{\mathrm{f}}, t_{\mathrm{i}}\right)\left|\psi\left(t_{\mathrm{i}}\right)\right\rangle
$$

we finally find the LZS propagator

$$
U_{\mathrm{LZS}}\left(t_{\mathrm{f}}, t_{\mathrm{i}}\right)=\left(\begin{array}{ll}
u_{11}\left(t_{\mathrm{f}}, t_{\mathrm{i}}\right) & u_{12}\left(t_{\mathrm{f}}, t_{\mathrm{i}}\right) \\
u_{21}\left(t_{\mathrm{f}}, t_{\mathrm{i}}\right) & u_{22}\left(t_{\mathrm{f}}, t_{\mathrm{i}}\right)
\end{array}\right)
$$


with

$$
\begin{aligned}
& u_{11}\left(t_{\mathrm{f}}, t_{\mathrm{i}}\right)=u_{22}^{*}\left(t_{\mathrm{f}}, t_{\mathrm{i}}\right)= \\
& \frac{\Gamma\left(1-\frac{\mathrm{i} \eta^{2}}{2}\right)}{\sqrt{2 \pi}}\left[D_{\frac{\mathrm{i} \eta^{2}}{2}}\left(\sqrt{2} \mathrm{e}^{-\frac{\mathrm{i} \pi}{4}} \tau_{\mathrm{f}}\right) D_{\frac{\mathrm{i} \eta^{2}}{2}-1}\left(\sqrt{2} \mathrm{e}^{\frac{3 \mathrm{i} \pi}{4}} \tau_{\mathrm{i}}\right)\right. \\
& \left.+D_{\frac{\mathrm{i} \eta^{2}}{2}}\left(\sqrt{2} \mathrm{e}^{\frac{3 \mathrm{i} \pi}{4}} \tau_{\mathrm{f}}\right) D_{\frac{\mathrm{i} \eta^{2}}{2}-1}\left(\sqrt{2} \mathrm{e}^{-\frac{\mathrm{i} \pi}{4}} \tau_{\mathrm{i}}\right)\right]
\end{aligned}
$$

and

$$
\begin{aligned}
& u_{12}\left(t_{\mathrm{f}}, t_{\mathrm{i}}\right)=-u_{21}^{*}\left(t_{\mathrm{f}}, t_{\mathrm{i}}\right)= \\
& \frac{\Gamma\left(1-\frac{\mathrm{i} \eta^{2}}{2}\right)}{\sqrt{\pi} \eta} \mathrm{e}^{\frac{\mathrm{i} \pi}{4}}\left[-D_{\frac{\mathrm{i} \eta^{2}}{2}}\left(\sqrt{2} \mathrm{e}^{-\frac{\mathrm{i} \pi}{4}} \tau_{\mathrm{f}}\right) D_{\frac{\mathrm{i} \eta^{2}}{2}}\left(\sqrt{2} \mathrm{e}^{\frac{3 \mathrm{i} \pi}{4}} \tau_{\mathrm{i}}\right)\right. \\
& \left.+D_{\frac{\mathrm{i} \eta^{2}}{2}}\left(\sqrt{2} \mathrm{e}^{\frac{3 \mathrm{i} \pi}{4}} \tau_{\mathrm{f}}\right) D_{\frac{\mathrm{i} \eta^{2}}{2}}\left(\sqrt{2} \mathrm{e}^{-\frac{\mathrm{i} \pi}{4}} \tau_{\mathrm{i}}\right)\right] .
\end{aligned}
$$

In the original LZS problem $t=0$ is defined at the energy levels crossing. A situation where $t_{\mathrm{i}}<0$ and $t_{\mathrm{f}}>0$ corresponds to drive the system through the avoided crossing. The case $t_{\mathrm{i}}<0$ and $t_{\mathrm{f}}<0$ corresponds to stop the system before it goes through the avoided crossing. Finally, $t_{\mathrm{i}}>0$ and $t_{\mathrm{f}}>0$ corresponds to a system which is initially prepared after the avoided crossing.

\section{Appendix B: Asymptotic expansion of the parabolic cylinder functions}

The expression of the LZS propagator can be expressed with simpler functions when the argument $\tau \gg 1$ and the parameter $\eta \gg 1$, in this case the parabolic cylinder functions can be expanded asymptotically 30 . The necessary asymptotic forms to expand Eqs. (A16) and (A17) are

$$
\begin{gathered}
D_{\frac{ \pm \mathrm{i} \eta^{2}}{2}}\left(\sqrt{2} \mathrm{e}^{\mp \frac{\mathrm{i} \pi}{4}} \tau\right) \simeq \cos \theta \mathrm{e}^{\frac{\pi \eta^{2}}{8} \pm \mathrm{i} \xi} \\
D_{\frac{ \pm \mathrm{i} \eta^{2}}{2}-1}\left(\sqrt{2} \mathrm{e}^{\frac{\mp \mathrm{i} \pi}{4}} \tau\right) \simeq \frac{\sqrt{2}}{\omega} \sin \theta \mathrm{e}^{\frac{\pi \eta^{2}}{8} \pm \mathrm{i}\left(\xi+\frac{\pi}{4}\right)}, \\
D_{\frac{\mathrm{i} \eta^{2}}{2}}\left(\sqrt{2} \mathrm{e}^{\frac{3 \mathrm{i} \pi}{4}} \tau\right) \simeq \cos \theta \mathrm{e}^{-\frac{3 \pi \eta^{2}}{8}+\mathrm{i} \xi} \\
+\frac{\eta \sqrt{\pi}}{\Gamma\left(1-\frac{\mathrm{i} \eta^{2}}{2}\right)} \sin \theta \mathrm{e}^{-\frac{\pi \eta^{2}}{8}-\mathrm{i}\left(\xi+\frac{\pi}{4}\right)} \\
D_{\frac{\mathrm{i} \eta^{2}}{2}-1}\left(\sqrt{2} \mathrm{e}^{\frac{3 \mathrm{i} \pi}{4}} \tau\right) \simeq \frac{\sqrt{2}}{\eta} \sin \theta \mathrm{e}^{-\frac{3 \pi \eta^{2}}{8}+\mathrm{i}\left(\xi-\frac{3 \pi}{4}\right)} \\
+\frac{\sqrt{2 \pi}}{\Gamma\left(1-\frac{\mathrm{i} \eta^{2}}{2}\right)} \cos \theta \mathrm{e}^{-\frac{\pi \eta^{2}}{8}-\mathrm{i} \xi}
\end{gathered}
$$

where we have defined

$$
\xi=-\frac{\eta^{2}}{4}+\frac{\eta^{2}}{2} \ln \left(\frac{1}{\sqrt{2}}\left(\tau+\sqrt{\tau^{2}+\eta^{2}}\right)\right)+\frac{\tau}{2} \sqrt{\tau^{2}+\eta^{2}},
$$

and

$$
\begin{aligned}
& \cos \theta=\sqrt{\frac{1}{2}\left(1+\frac{\tau}{\sqrt{\tau^{2}+\eta^{2}}}\right)}, \\
& \sin \theta=\sqrt{\frac{1}{2}\left(1-\frac{\tau}{\sqrt{\tau^{2}+\eta^{2}}}\right)} .
\end{aligned}
$$

Using the above expressions and writing $\tau_{\mathrm{i}}=\mathrm{e}^{\mathrm{i} \pi}\left|\tau_{\mathrm{i}}\right|$ to fulfill the condition for the expansion we find,

$$
\begin{aligned}
& \begin{array}{l}
u_{11}\left(t_{\mathrm{f}}, t_{\mathrm{i}}\right)= \\
\sqrt{1-\mathrm{e}^{-\pi \eta^{2}}}\left(\sin \theta_{\mathrm{f}} \cos \theta_{\mathrm{i}} \mathrm{e}^{-\mathrm{i}\left(\xi_{\mathrm{f}}+\xi_{\mathrm{i}}+\arg \Gamma\left(1-\frac{\mathrm{i} \eta^{2}}{2}\right)+\frac{\pi}{4}\right)}\right. \\
\left.\quad+\sin \theta_{\mathrm{i}} \cos \theta_{\mathrm{f}} \mathrm{e}^{\mathrm{i}\left(\xi_{\mathrm{f}}+\xi_{\mathrm{i}}+\arg \Gamma\left(1-\frac{\mathrm{i} \eta^{2}}{2}\right)+\frac{\pi}{4}\right)}\right) \\
+\mathrm{e}^{-\frac{\pi \eta^{2}}{2}}\left(\cos \theta_{\mathrm{f}} \cos \theta_{\mathrm{i}} \mathrm{e}^{\mathrm{i}\left(\xi_{\mathrm{f}}-\xi_{\mathrm{i}}\right)}-\sin \theta_{\mathrm{f}} \sin \theta_{\mathrm{i}} \mathrm{e}^{-\mathrm{i}\left(\xi_{\mathrm{f}}-\xi_{\mathrm{i}}\right)}\right),
\end{array}
\end{aligned}
$$

and

$$
\begin{aligned}
& u_{12}\left(t_{\mathrm{f}}, t_{\mathrm{i}}\right)=-u_{21}^{*}\left(t_{\mathrm{f}}, t_{\mathrm{i}}\right) \simeq \\
& \sqrt{1-\mathrm{e}^{-\pi \eta^{2}}}\left(\sin \theta_{\mathrm{f}} \sin \theta_{\mathrm{i}} \mathrm{e}^{-\mathrm{i}\left(\xi_{\mathrm{f}}+\xi_{\mathrm{i}}+\arg \Gamma\left(1-\frac{\mathrm{i} \eta^{2}}{2}\right)+\frac{\pi}{4}\right)}\right. \\
& \left.\quad-\cos \theta_{\mathrm{f}} \cos \theta_{\mathrm{i}} \mathrm{e}^{\mathrm{i}\left(\xi_{\mathrm{f}}+\xi_{\mathrm{i}}+\arg \Gamma\left(1-\frac{\mathrm{i} \eta^{2}}{2}\right)+\frac{\pi}{4}\right)}\right) \\
& +\mathrm{e}^{-\frac{\pi \eta^{2}}{2}}\left(\cos \theta_{\mathrm{i}} \sin \theta_{\mathrm{f}} \mathrm{e}^{-\mathrm{i}\left(\xi_{\mathrm{f}}-\xi_{\mathrm{i}}\right)}+\cos \theta_{\mathrm{f}} \sin \theta_{\mathrm{i}} \mathrm{e}^{\mathrm{i}\left(\xi_{\mathrm{f}}-\xi_{\mathrm{i}}\right)}\right) .
\end{aligned}
$$

where $\cos \theta_{\mathrm{i}, \mathrm{f}}, \sin \theta_{\mathrm{i}, \mathrm{f}}$, and $\xi_{\mathrm{i}, \mathrm{f}}$ are respectively given by Eq. (B6) and Eq. (B5) for $\tau=\mathrm{e}^{\mathrm{i} \pi}\left|\tau_{\mathrm{i}}\right|, \tau_{\mathrm{f}}$.

We noticed that the asymptotic expansions (B1), (B2), (B3), and (B14) are valid for the weaker condition $\tau+\eta \gg$ 1 , as already reported in Ref. 15 for the expansion of $\left|u_{11}\right|^{2}$ and $\left|u_{12}\right|^{2}$.

\section{Appendix C: The LZS propagator as a rotation}

In quantum mechanics the rotation operator $\mathcal{D}(\hat{n}, \vartheta)$ by an angle $\vartheta$ around an axis $\hat{n}$ of a two-level system has the representation

$$
\begin{aligned}
\mathcal{D}(\hat{n}, \vartheta) & =\mathrm{e}^{\mathrm{i} \hat{n} \cdot \sigma \frac{\vartheta}{2}} \\
& =\left(\begin{array}{ll}
\cos \frac{\vartheta}{2}-\mathrm{i} n_{z} \sin \frac{\vartheta}{2} & \left(-\mathrm{i} n_{x}-n_{y}\right) \sin \frac{\vartheta}{2} \\
\left(-\mathrm{i} n_{x}+n_{y}\right) \sin \frac{\vartheta}{2} & \cos \frac{\vartheta}{2}+\mathrm{i} n_{z} \sin \frac{\vartheta}{2}
\end{array}\right) .
\end{aligned}
$$



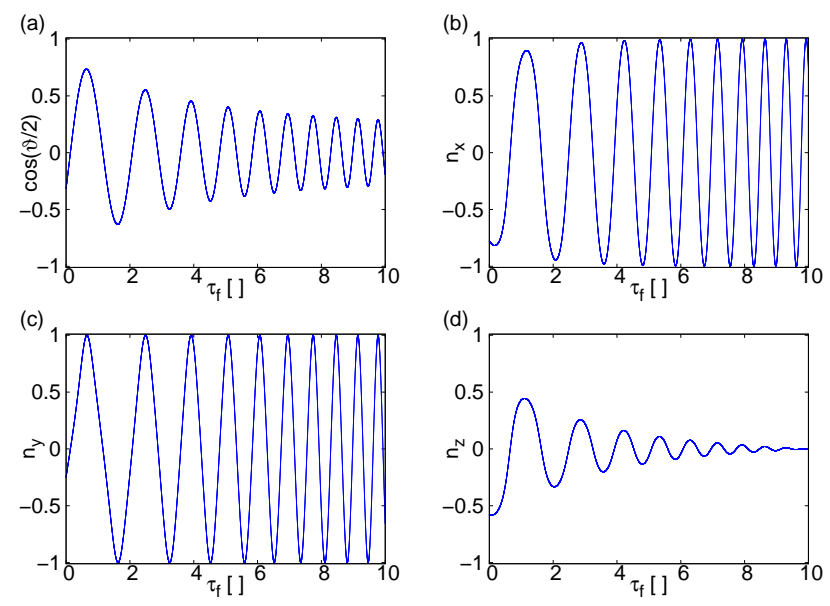

Figure 6. (color online) (a) Cosine of the rotation angle and components of the rotation axis (b), (c), and (d) as a function of $\tau_{\mathrm{f}}$ for the dimensionless parameters $\tau_{\mathrm{i}}=10$ and $\eta=3$.

Identifying Eqs. (C1) and (A15) with $u_{i j}$ given by Eqs. (B7) and (B8) we can express the rotation angle $\vartheta$ and the rotation axis $\hat{n}$ as functions of the LZS propagator parameters $\tau_{\mathrm{i}}, \tau_{\mathrm{f}}, \eta$. We have

$$
\begin{aligned}
\cos \frac{\vartheta}{2}= & \sqrt{1-\mathrm{e}^{-\pi \eta^{2}}} \cos \zeta\left[\sin \theta_{\mathrm{f}} \cos \theta_{\mathrm{i}}+\cos \theta_{\mathrm{f}} \sin \theta_{\mathrm{i}}\right] \\
& +\mathrm{e}^{-\frac{\pi \eta^{2}}{2}} \cos \varsigma\left[\cos \theta_{\mathrm{f}} \cos \theta_{\mathrm{i}}-\sin \theta_{\mathrm{f}} \sin \theta_{\mathrm{i}}\right]
\end{aligned}
$$

$$
\zeta=\xi_{\mathrm{f}}+\xi_{\mathrm{i}}+\arg \Gamma\left(1-\frac{\mathrm{i} \eta^{2}}{2}\right)+\frac{\pi}{4}
$$

and

$$
\varrho=\xi_{\mathrm{f}}-\xi_{\mathrm{i}} .
$$

The components of the rotation axis are given by (see Fig. 6)

$$
\begin{aligned}
n_{x}=\frac{1}{\sin \frac{\vartheta}{2}} & \left(\sqrt{1-\mathrm{e}^{-\pi \eta^{2}}} \sin \zeta\left[\sin \theta_{\mathrm{f}} \sin \theta_{\mathrm{i}}+\cos \theta_{\mathrm{f}} \cos \theta_{\mathrm{i}}\right]\right. \\
& \left.+\mathrm{e}^{-\frac{\pi \eta^{2}}{2}} \sin \varrho\left[\cos \theta_{\mathrm{i}} \sin \theta_{\mathrm{f}}-\cos \theta_{\mathrm{f}} \sin \theta_{\mathrm{i}}\right]\right),
\end{aligned}
$$

$$
\begin{array}{r}
n_{y}=\frac{1}{\sin \frac{\vartheta}{2}}\left(\sqrt{1-\mathrm{e}^{-\pi \eta^{2}}} \cos \zeta\left[-\sin \theta_{\mathrm{f}} \sin \theta_{\mathrm{i}}+\cos \theta_{\mathrm{f}} \cos \theta_{\mathrm{i}}\right]\right. \\
\left.+\mathrm{e}^{-\frac{\pi \eta^{2}}{2}} \cos \varrho\left[-\cos \theta_{\mathrm{i}} \sin \theta_{\mathrm{f}}-\cos \theta_{\mathrm{f}} \sin \theta_{\mathrm{i}}\right]\right), \\
n_{z}=\frac{1}{\sin \frac{\vartheta}{2}}\left(\sqrt{1-\mathrm{e}^{-\pi \eta^{2}}} \sin \zeta\left[\sin \theta_{\mathrm{f}} \cos \theta_{\mathrm{i}}-\cos \theta_{\mathrm{f}} \sin \theta_{\mathrm{i}}\right]\right. \\
\left.+\mathrm{e}^{-\frac{\pi \eta^{2}}{2}} \sin \varrho\left[-\cos \theta_{\mathrm{f}} \cos \theta_{\mathrm{i}}+\sin \theta_{\mathrm{f}} \sin \theta_{\mathrm{i}}\right]\right) .
\end{array}
$$

where

88, 186802 (2002).

18 W. A. Coish and D. Loss, Phys. Rev. B 72, 125337 (2005).

19 H. Ribeiro and G. Burkard, Phys. Rev. Lett. 102, 216802 (2009).

20 D. Vion et al., Science 296, 886 (2002).

21 T. Hayashi et al., Phys. Rev. Lett. 91, 226804 (2003).

22 R. Hanson and G. Burkard, Phys. Rev. Lett. 98, 050502 (2007)

23 J. M. Taylor et al., Nature Physics 1, 177 (2005).

24 D. Stepanenko and G. Burkard, Phys. Rev. B 75, 085324 (2007).

25 M. Pioro-Ladrière et al., Nature Phys. 4, 776 (2008).

26 J. R. Petta et al., Phys. Rev. Lett. 100, 067601 (2008).

27 D. Klauser, W. A. Coish, and D. Loss, Phys Rev. B 73, 205302 (2006).

28 D. Stepanenko et al., Phys. Rev. Lett. 96, 136401 (2006).

29 M. Abramowitz and I. A. Stegun, Handbook of Mathematical Functions, Ch. 19, Dover (1970).

${ }^{30}$ F. W. J. Olver, J. Res. Natl. Bur. Stand. 63B, 131 (1959).

16 Y. Kayanuma and S. Fukuchi, J. Phys. B 18, 4089 (1985).

17 A. V. Khaetskii, D. Loss, and L. Glazman, Phys. Rev. Lett. 